\title{
вмJ Global Health Field detection devices for screening the quality of medicines: a systematic review
}

\author{
Serena Vickers, ${ }^{1,2,3}$ Matthew Bernier, ${ }^{4,5}$ Stephen Zambrzycki, ${ }^{4}$ \\ Facundo M Fernandez, ${ }^{4}$ Paul N Newton, ${ }^{1,2,3}$ Céline Caillet ${ }^{1,2,3}$
}

To cite: Vickers S, Bernier M, Zambrzycki S, et al. Field detection devices for screening the quality of medicines: a systematic review. BMJ Glob Health 2018:3:e000725. doi:10.1136/ bmjgh-2018-000725

Handling editor Seye Abimbola

- Additional material is published online only. To view please visit the journal online (http://dx.doi.org/10.1136/ bmjgh-2018-000725).

Received 17 January 2018 Revised 20 June 2018 Accepted 24 June 2018

Check for updates

Cㄱ Author(s) (or their employer(s)) 2018. Re-use permitted under CC BY. Published by BMJ.

${ }^{1}$ Lao-Oxford-Mahosot HospitalWellcome Trust Research Unit (LOMWRU), Microbiology Laboratory, Mahosot Hospital, Vientiane, Laos

${ }^{2}$ Centre for Tropical Medicine and Global Health, University of Oxford, Oxford, UK

${ }^{3}$ Infectious Diseases Data Observatory (IDD0)/Worldwide Antimalarial Resistance Network (WWARN), University of Oxford, Oxford, UK

${ }^{4}$ School of Chemistry and Biochemistry, Georgia Institute of Technology, Atlanta, Georgia, USA

${ }^{5}$ Campus Chemical Instrument Center Mass Spectrometry and Proteomics Facility, The Ohio State University, Columbus, Ohio, USA

Correspondence to

Dr Céline Caillet;

celine.caillet@iddo.org

\section{ABSTRACT}

Background Poor quality medicines have devastating consequences. A plethora of innovative portable devices to screen for poor quality medicines has become available, leading to hope that they could empower medicine inspectors and enhance surveillance. However, information comparing these new technologies is woefully scarce. Methods We undertook a systematic review of Embase, PubMed, Web of Science and SciFinder databases up to 30 April 2018. Scientific studies evaluating the performances/ abilities of portable devices to assess any aspect of the quality of pharmaceutical products were included. Results Forty-one devices, from small benchtop spectrometers to 'lab-on-a-chip' single-use devices, with prices ranging from $<$ US $\$ 10$ to $>$ US $\$ 20000$, were included. Only six devices had been field-tested (GPHFMinilab, CD3/CD3+, TruScan RM, lateral flow dipstick immunoassay, CBEx and Speedy Breedy). The median (range) number of active pharmaceutical ingredients (APIs) assessed per device was only $2(1-20)$. The majority of devices showed promise to distinguish genuine from falsified medicines. Devices with the potential to assay API (semi)-quantitatively required consumables and were destructive (GPHF-Minilab, PharmaChk, aPADs, lateral flow immunoassay dipsticks, paper-based microfluidic strip and capillary electrophoresis), except for spectroscopic devices. However, the 10 spectroscopic devices tested for their abilities to quantitate APIs required processing complex API-specific calibration models. Scientific evidence of the ability of the devices to accurately test liquid, capsule or topical formulations, or to distinguish between chiral molecules, was limited. There was no comment on cost-effectiveness and little information on where in the pharmaceutical supply chain these devices could be best deployed.

Conclusion Although a diverse range of portable field detection devices for medicines quality screening is available, there is a vitally important lack of independent evaluation of the majority of devices, particularly in field settings. Intensive research is needed in order to inform national medicines regulatory authorities of the optimal choice of device(s) to combat poor quality medicines.

\section{INTRODUCTION}

According to a recent $\mathrm{WHO}$ report, $~ 10 \%$ of medical products circulating in low-income and middle-income countries (L/MICs) are either substandard or falsified. ${ }^{1}$ Although this

\section{Key questions}

What is already known?

- $10 \%$ of medical products circulating in low-income and middle-income countries are either substandard or falsified, leading to increased morbidity and mortality, adverse drug reactions, economic losses and diminished public confidence in health systems.

- A large number of portable screening devices have recently been developed that could aid medicines regulatory authorities in the detection of poor quality medicines, but there is scanty evidence to inform policy makers as to which device to use and where.

What are the new findings?

- Forty-one devices covering 19 technologies were identified; more than half of these devices employed spectroscopic techniques.

- Field evaluation has been published for only 6 of 41 devices and

- ignificant knowledge gaps exist, impairing evidence-based policy decisions.

What do the new findings imply?

- There is inadequate independent evaluation of these devices to inform policy makers about optimal choice of device to combat poor quality medicines.

- Intensive research is needed to understand the comparative advantages and limitations of the different devices and technologies.

problem is as old as the medicinal trade, ${ }^{23}$ its impact on global health has been largely under-recognised. L/MICs are significantly affected, ${ }^{4-6}$ but wealthier countries with good regulatory systems are not immune. ${ }^{7-9}$ Substandard and falsified (SF) medicines (box 1) have devastating consequences, including increased morbidity and mortality, economic losses and diminished public confidence in health systems. Poor quality antimicrobials, particularly those containing reduced quantities of active pharmaceutical ingredients (APIs), may be a key but neglected driver of antimicrobial resistance (AMR). ${ }^{10}$ Despite this, the oversight and penalties for perpetrators are weak, and falsifying medicines remains an attractive criminal activity. ${ }^{11}$ 
Box 1 Definitions of substandard and falsified medicines $^{86}$

- Substandard, also called 'out of specification': authorised medical products that fail to meet either their quality standards or specifications, or both.

- Falsified: medical products that deliberately/fraudulently misrepresent their identity, composition or source.

Medicines regulatory authorities (MRAs) are responsible for preventing, detecting and removing SF medicines. Others actors involved in medicine procurement (eg, non-governmental organisations, procurement agencies and hospital pharmacies) are, together with MRAs, keystones for the majority of potential interventions to prevent, detect and remove poor quality medicines. Currently, in L/MICs these key actors often have only their own senses and knowledge to rely on as they seek circulating SF medicines. Samples may then be sent for formal chemical analysis laboratory testing, using API-specific (and dosage formulation-specific) validated pharmacopeial protocols, or non-validated inhouse procedures when pharmacopeial methods do not exist. However, these tests (such as high-performance liquid chromatography (HPLC)) are expensive, time-consuming and scarce in many countries (box 2). There are often significant delays between collection of suspicious medicines and confirmation of their poor quality, with harm spreading unchecked in the interim.

Over the last two decades a plethora of portable medicine analysis screening tools have been developed, offering the potential for objective analysis of medicines in the 'field'. A previous review compared the suitability of different existing chemical analysis technologies for $\mathrm{L} / \mathrm{MICs}^{12}$ (eg, Raman spectroscopy, colourimetry). With more devices and more data now available, we undertook a systematic review to understand the performance and characteristics of portable devices for the field evaluation of medicines. This review identifies multiple gaps in the evidence for optimal device selection to inform policy decisions on which devices to use to screen medicine quality before sending samples for confirmatory analyses, where and when.

\section{METHODS}

\section{Search strategy and selection criteria}

A systematic review was conducted, following the Preferred Reporting Items for Systematic Reviews and Meta-Analyses guidelines (online supplementary file 1, PRISMA checklist) with registration in the international prospective register of systematic reviews (PROSPERO, ID 42016043216). We searched for English-language scientific articles on portable technologies used to assess the quality of pharmaceutical products, using Embase (from 1947), PubMed (from 1946), Web of Science (from 1900) and SciFinder (from 1840) to 30 April 2018. Search terms included those related to the equipment
Box 2 Main technologies used in pharmaceutical quality analysis

\section{Colourimetry}

- Colourimetric techniques use analysis of the colour developed by a sample in the presence of specific reagents. The presence or absence of the colour gives information on the presence or absence of the chemical compound (or specific chemical groups) being investigated. The intensity of the colour, interpreted either by the naked eye or by specific devices (called colourimeters or photometers), can provide quantitative information on the amount of the chemical within the medicine.

\section{Chromatography}

- This technology separates different ingredients in a mixture to obtain pure compounds to show their presence (or absence) and quantity. As many compounds are colourless, specific detectors are used to reveal them, such as those based on refraction index changes, fluorescence or absorbance at various wavelengths.

- Individual compounds are separated from each other through their interaction with a solid 'stationary phase', which remains fixed in a column or support. A liquid or a gas 'mobile phase' flows through the stationary phase and the captured compounds gradually move along the stationary phase in the same direction as the mobile phase. Each compound of a mixture will travel through the stationary phase, ejecting at varying times due to their different affinities with the stationary versus mobile phases. For examining medicines quality, the result from the test sample is compared with the result yielded by the authentic product, tested under the same conditions.

- Thin-layer chromatography uses a thin layer of silica or paper as the stationary phase. The mobile phase travels through the stationary phase via capillary action when the base of the device is placed with one end dipped in a solution. Once the device is pulled from the solution, the separation process stops and the separated compounds are retained spatially on the stationary phase, and revealed with the use of a lamp or chemical reagent.

- High-performance liquid chromatography forces the mobile phase through a column of stationary phase silica particles by high-pressure pumps. A detector monitors the compounds as they are released, allowing the identification of the compounds based on their specific retention times and quantitation based on their peak area. Typical detectors vary in terms of cost and specificity, and include UV-Vis light absorbance detectors and (quadrupole, ion trap, timeof-flight, orbitrap) mass spectrometers.

Spectroscopy: near-infrared (NIR), mid-infrared (MIR), Raman and ultraviolet-visible light (UV-Vis)

- Different chemicals have their own unique interaction with electromagnetic radiation. The type of interaction depends on the nature of the compound's molecular structure and the radiation used. When a sample is irradiated with a specific wavelength (energy) of light, structures within the sample absorb that energy and vibrate along different chemical bonds which can be measured by NIR, MIR and Raman spectroscopy, types of 'vibrational spectroscopy'. These 'vibrations' cause the absorbance or emittance of light by the sample in a characteristic spectrum, unique to the sample-often called a 'spectral fingerprint'. Usually, this unique spectrum has to undergo mathematical transformation (spectral processing) to be readable by the user. In order to identify whether a sample is authentic or substandard/falsified, the sample spectrum generated is compared with the spectrum of the authentic product to assess its similarity.

Continued 


\section{Box 2 Continued}

This requires the construction of a 'reference library' or database consisting of the spectra of authentic products.

- UV-Vis spectroscopy uses light within the ultraviolet and visible regions of the electromagnetic spectrum. UV-Vis absorbance measurements, which monitor the amount of light within this part of the spectrum that is transmitted through a material, do not reveal as much structural information as NIR, MIR and Raman. However, fluorescence and luminescence signals can be measured within the UV-Vis region. Signals from samples that can emit UV-Vis light through fluorescence (ie, the sample can be excited by a wavelength of light and then emit a different wavelength of light) and luminescence (a chemical reaction emits light from the sample) can thus be used to characterise and quantify the amount of active pharmaceutical ingredients within a sample.

\section{Structurally based separation techniques}

- Molecules of different mass and charge move differently when under the influence of an external electric field (heavier molecules travel slower or require stronger electric fields to be transported). These travel times or electric field conditions are recorded by a detector and are correlated to the mass and charge of the molecule, allowing its identification. Mass spectrometry, ion mobility spectroscopy and capillary electrophoresis all exploit this phenomenon. Mass spectrometry measures movement through a vacuum, ion mobility in the gas phase and capillary electrophoresis in the liquid phase. For examining medicines quality, the result from the test sample is compared with the result yielded by the authentic product, tested under the same conditions.

(eg, 'device', 'instrument'), terms referring to the portability of the equipment (eg, 'portable', 'handheld') and terms related to the quality of pharmaceutical products (eg, 'substandard', 'falsified'). The full search strategies are provided in online supplementary file 2.

After removal of duplicates, titles and abstracts were independently screened for eligibility by two authors (SV, $\mathrm{MB})$. Any reservations on eligibility for inclusion were resolved by discussion between the three reviewers ( $\mathrm{SV}$, MB, CC), with final adjudication from FMF. References in English and French provided by colleagues working in the field, in addition to references within reviews of specific techniques, and those in all included articles, were examined to identify additional relevant articles. We included all studies evaluating the performances/abilities of portable devices to assess any aspect of the quality of pharmaceutical products in a laboratory environment, in field surveys and proof-of-concept articles in which the authors stress the potential portability of a method. Studies with the aim to estimate medicine quality prevalence were only included if theycontained information on the performances/abilities of the device as a portable technology for field use . Devices currently under development (although not yet marketed) and devices no longer marketed but superseded by other devices were included. Non-portable devices, devices used for testing the quality of non-pharmaceutical products or for identification of traditional medicines, devices for measuring APIs in biological fluids, and product security technologies were excluded. Patent application publications, articles on the development of a method (eg, a new thin-layer chromatography (TLC) method) not intended for deployment in a field detection kit, reviews/general discussions and articles describing or comparing methods for spectral analysis (chemometrics) rather than the performance of the device itself were also excluded.

CC, MB and SV independently reviewed and extracted data from the eligible articles. For included devices, additional information on objective characteristics (eg, physical appearance, approximate cost and market status) was obtained from manufacturers' websites and enquiries to them.

\section{Key variables and definitions}

In this review, 'portable' refers to transportable equipment (ie, intended to be moved from one place to another whether or not connected to a main electrical supply ${ }^{13}$ ) able to be carried by a maximum of two persons and that requires minimal set-up on arrival at the field detection site (set-up can be managed by technician-level staff after short training on the device). Devices that require an initial laboratory phase set-up from highly trained staff (eg, Raman spectrometers which require creation of reference libraries and complex processing of spectral data) but that are subsequently portable and easy to use in the field by technician-level staff were included. 'Field-tested' device refers to a device assessed onsite, that is, near where the medicines were collected, as opposed to formal laboratory-based studies. A reference standard refers to a specimen of the medicine API intended for use in compendial methods, which is of the highest possible purity and highly characterised by analytical chemistry techniques, used as a direct chemical comparator or to generate a signature. ${ }^{14} \mathrm{~A}$ reference library refers to a library of measurements of authentic medicines collected by the device and with which the device compares the measurement obtained from a test sample, most commonly spectral libraries of authentic measurements stored within the spectrometer software ('Spectral Reference Library'). Semi-quantitative is defined as an approximate measurement of the amount of a substance, between a qualitative and a quantitative result (eg, between $80 \%$ and $100 \%$ of the stated amount). 'Non-destructive' refers to devices used to test intact dosage units of medicines (predominantly tablets) either through packaging or without perturbing the dosage unit.

Sensitivity is defined as the proportion of medicines that are detected as poor quality by the device out of all the medicines determined as poor quality by a reference technique. Specificity is defined as the proportion of medicines that are identified as authentic by the device out of all the medicines determined as good quality by a reference technique. 


\section{Data analysis}

Data were extracted and entered into a Microsoft Excel spreadsheet. For each device, the developer's names, type of technology used, main technical specifications (eg, resolution, spectral range), reported sensitivity, specificity and other laboratory or field test results, practical aspects of the use of the device (eg, the measurement time per sample, consumables required), and the pluses and minuses quoted by the authors were extracted when available. For clarity, we have presented only the key results from devices tested on finished pharmaceutical products and only when poor quality medicines (either field-collected or simulated products) were used for evaluation. The quality of the included studies could not be objectively assessed because of the wide heterogeneity of study designs and a lack of consensus guidelines for reporting.

\section{RESULTS}

Of the 5718 reports screened, 282 full-text papers were assessed for eligibility (online supplementary file 3, PRISMA flow diagram). Of these, 62 matched the inclusion criteria and were included in the review.

Forty-one devices were identified in the 62 articles (table 1, figure 1).

All evaluations were performed in a laboratory setting unless stated otherwise. We classify devices into those (1) that examine the pharmaceutical formulation, that is, both API(s) and excipients present in the finished pharmaceutical product ('formulation screening'); (2) those which focus on API(s) detection only; (3) those 'Physical Analysis Devices', which primarily assess the physical, rather than chemical, properties of samples; and (4) those that have the ability to detect microbial contamination.

A summary of the reference requirements per device is available in online supplementary file 4 , and all the extracted information is presented in online supplementary files 5 and 6.

\section{Formulation screening devices}

The devices in this section examine the chemical 'fingerprint' of a formulation (both $\mathrm{API}(\mathrm{s})$ and excipients) and are classified by whether they have been tested for their ability to perform quantitative API analysis or not. The ability of these devices to discriminate between poor quality and good-quality medicines, and to quantitate APIs, depends on both the performance of the device and on the postacquisition processing of spectral data by the associated software. All require a spectral reference library, but are typically non-destructive and do not require consumables.

\section{Devices tested for their ability to do quantitative analysis of APIs}

As far as we are aware, the devices tested for their abilities to quantitate APIs do not currently have inbuilt software to provide quantitative results. The performance results regarding quantitative abilities of the devices presented in this section were obtained after data processing within the laboratory settings was performed.

The Raman TruScan RM (Thermo Scientific) is one of the six devices tested in the field (table 2). It discriminated between 14 poor quality (falsified and degraded medicines) and 70 authentic antimalarials with $100 \%$ sensitivity and $99 \%$ specificity. ${ }^{15}$ Forty-four falsified samples (of 8 different products) and 62 formulations of genuine products (unstated APIs, 33 'product families' in total) were identified with $100 \%$ accuracy. ${ }^{16}$ The TruScan showed similar match/fail performance for medicines identification (despite lower signal resolution) when compared with Raman benchtop instruments. ${ }^{16-18}$

After applying a sample preparation method, special agents in a mail facility tested 14 samples of Viagra (12 contained sildenafil) with the TruScan RM with an accuracy of $91.7 \%$ to qualitatively identify the presence/absence of sildenafil (Fourier Transform-Infrared (FT-IR) analysis as reference technique). ${ }^{19}$ Different strengths (simulating 'substandard' medicines) of the same antimalarial APIs and brand could not be reliably distinguished using the match/not-match approach. ${ }^{1520}$ In addition, one in three placebos wrongly passed the identification test versus their full API strength counterpart. ${ }^{16}$ In investigations of the $p$ values (threshold limit for a sample to give a 'pass' or 'fail') obtained by scanning five products containing candesartan with the TruScan RM, it was suggested that the $p$ value could be set at 0.40 (instead of 0.05 ) for the device to better discriminate substandard medicines containing less than $50 \%$ or more than $150 \%$ API from the good-quality products. ${ }^{21}$ However, these results should be taken with caution considering the small sample size. The FirstDefender TruScan (superseded by the TruScan RM) determined the amount of APIs to within $1.6 \%-12 \%$ of the reference assay for experimental finished products of acetylsalicylic acid, ascorbic acid and caffeine. ${ }^{22}$

The Metrohm Instant Raman Analyzer (MIRA, Metrohm) discriminated between different concentrations of injectable doxorubicin $(n=90)$ and epirubicin $(n=90)$ through glass containers with $100 \%$ sensitivity and specificity. Quantitation with a coefficient of determination $\left(r^{2}\right)$ of 0.99 was reported. ${ }^{23}$

The EZRaman-I (TSI) qualitatively confirmed the presence of the stated API in four finished drug products containing amoxicillin, acyclovir and doxycycline ${ }^{24}$ with a Raman binary barcode method using a reference library containing Raman spectra of APIs. ${ }^{25}$ On averaging the results of the per cent of the API claimed on the label of five tablets per sample, predictions by the EZRaman-I were within $3 \%$ of the HPLC results for three out of four products, and one product showed a value of $6.0 \%$ lower than those obtained by HPLC.

Among the 14 devices based on Infrared (IR)/Mid-Infrared (Mid-IR), the MicroPhazir (and its predecessor the Phazir) has been tested on small sample sets of different types of raw or finished pharmaceutical product types in the laboratory. ${ }^{22}$ 26-33 Spectral data have been successfully 


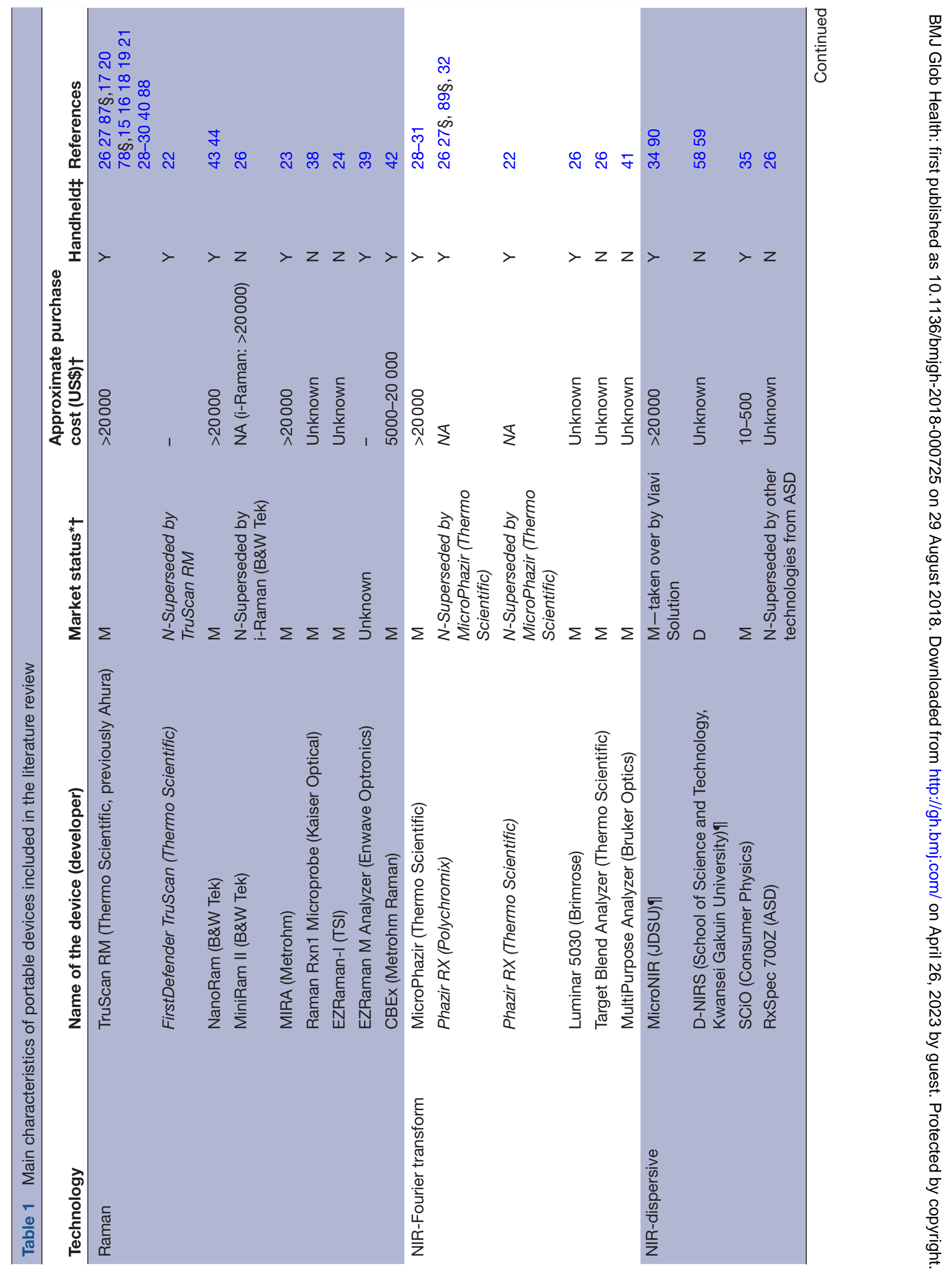


acquired through transparent blister packaging using six experimental samples. ${ }^{28}$ Quantitation of acetylsalicylic acid, ascorbic acid and caffeine in tablets gave results comparable with a reference benchtop Fourier Transform-Near-Infrared (FT-NIR) instrument. ${ }^{22}$

The MicroNIR (JDSU, then Viavi) and the TruDefender FT (Thermo Scientific) have been used for quantitation of API to within $0.1 \%-7.8 \%$ of the reference for weight loss and erectile dysfunction medicines, and also for acetylsalicylic acid, ascorbic acid and caffeine. ${ }^{22} 34$

The SCiO (Consumer Physics) device showed 100\% specificity and sensitivity in the identification of falsified $(n=42)$ versus genuine $(n=54)$ antimalarials, but failed to quantify the amount of amodiaquine in finished products. This device was able to quantify artesunate with $95 \%$ certainty in 15 oral products. ${ }^{35}$

The compact benchtop QDa single quadrupole mass spectrometer (Waters) correctly identified the Artemisinin-based combination therapy (ACT) artemether+lumefantrine and other compounds, such as chloramphenicol, ciprofloxacin and sugars, in 192 seized falsified antimalarials. The relative intensity of each compound detected could be compared from run to run between tablets and used as a proxy for API quantitation. ${ }^{36}$ For quantitation, the handheld Mini 10mass spectrometer has been demonstrated to produce parts-per-billion detection limits for drugs of abuse ${ }^{37}$ but has not yet been evaluated for poor quality medicines.

Devices with untested potential to perform quantitative analysis of APIS

Three Raman devices (MiniRam II (B\&W Tek), Raman Rxn1 Microprobe (Kaiser Optical) and EZRaman M Analyzer (Enwave Optronics) $)^{26} 3839$ and nine near-infrared/mid-infrared devices (MultiPurpose Analyzer (Bruker Optics), Luminar 5030 (Brimrose), RxSpec 700Z (ASD), Exoscan (A2 Technologies), MLp (A2 Technologies), FT/IR-4100 (JASCO), Cary 630 (Agilent), Nicolet iS10 (Thermo Scientific) and Target Blend Analyzer (Thermo Scientific)) were included in small-scale laboratory studies. ${ }^{26} 29304041$

Of note, the CBEx (Metrohm Raman) successfully identified the presence of paracetamol, amoxicillin, lumefantrine and pyrazinamide in various APIs/API combinations but failed to identify other APIs in fixeddrug combinations, (eg, artemether in artemether-lumefantrine tablets), and furosemide and oxytocin in single API injection samples. ${ }^{42}$ A limited set of artificially degraded samples were correctly identified with accuracies depending on the API and the level/type of degradation. A field evaluation of the utility of the device, rather than its performance (table 2), among 10 operators from the regulatory authorities of India and Zimbabwe with various technical experience, suggested this as a well-functioning device requiring less than 2 weeks of training. ${ }^{42}$

The NanoRam (B\&W Tek) showed 100\% sensitivity and 96\% specificity (against TLC, with HPLC used to confirm 


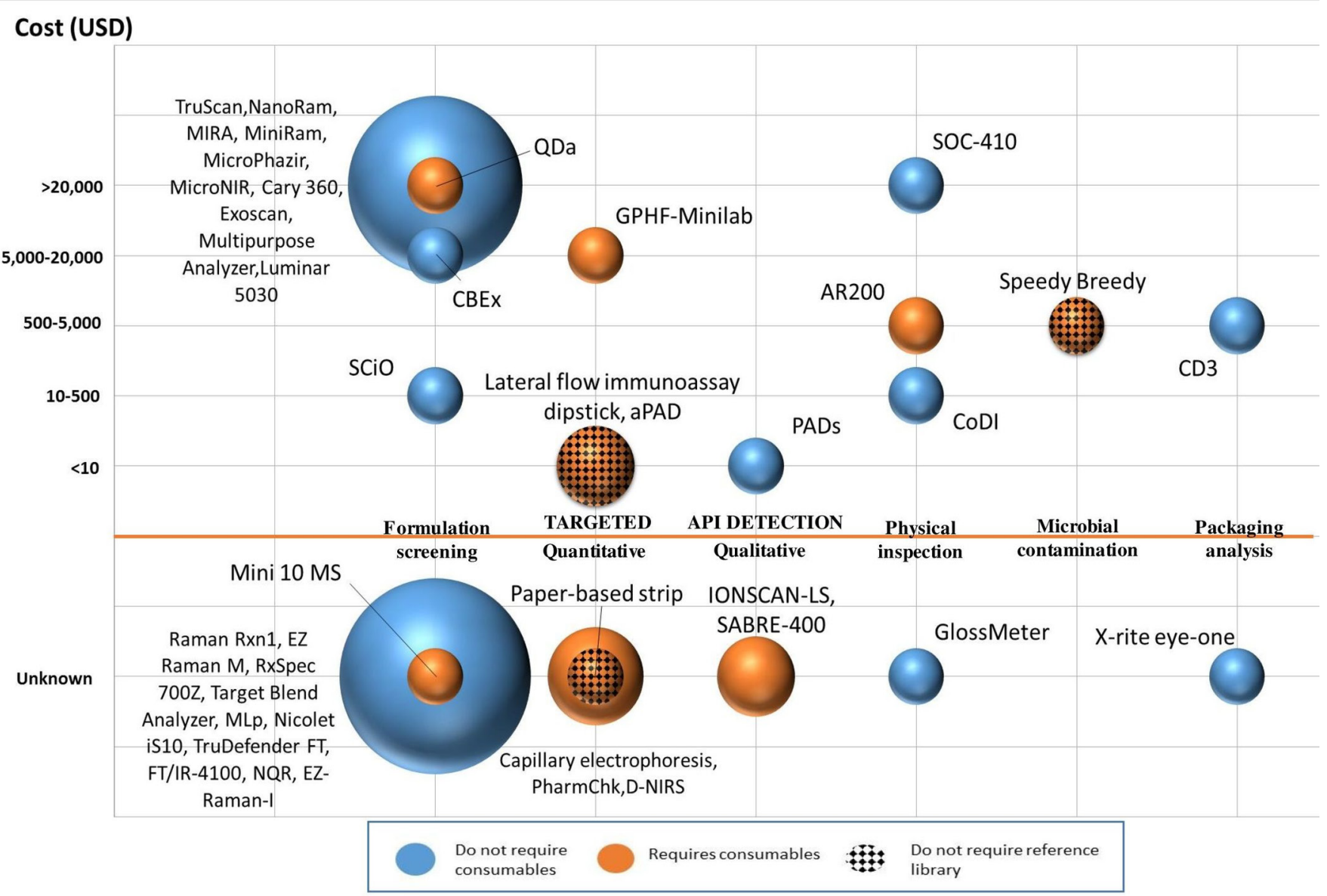

NB : aPADs are paper analytical devices able to semi-quantitate an API whereas the PADs are designed to qualitatively screen for the absence or presence of some APIs and excipients

Figure 1 Main characteristics of the included devices by type of analysis, cost at purchase, requirement for consumables and/or reference library. The size of the circles is proportional to the number of devices. APIs, active pharmaceutical ingredients; CoDI, Counterfeit Drug Indicator; PADs, paper analytical devices.

samples which failed TLC) in the investigation of 289 antimalarial samples (including 24 falsified and 22 experimental 'wrong API fakes' containing paracetamol). ${ }^{43} 44$

A prototype nuclear quadrupole resonance (NOR) device has been used successfully to identify ampicillin in capsules and paracetamol in tablets through their original packaging. No data on its sensitivity and specificity have yet been reported. ${ }^{45}$

\section{Targeted API detection devices}

Most devices in this section are semi-quantitative. All techniques require sample destruction and most require sample pretreatment (eg, dissolution). Some provide both qualitative and quantitative information and may provide data on other properties (eg, disintegration characteristics). Others simply qualitatively identify the API.

Quantitative and semi-quantitative targeted API detection devices The GPHF-Minilab (Global Pharma Health Fund) is a 'lab-in-a-suitcase' containing the supplies for visual physical inspection of the medicine (both dosage form and packaging), identification and semi-quantitation of the stated API by TLC, and disintegration testing. It is one of the six field-tested devices identified in this review (table 2).

In a field survey of 84 antimalarial medicines (including 14 substandard/falsified samples) in Ghana, $100 \%$ sensitivity and specificity were reported for Minilab TLC identification against HPLC reference assays. ${ }^{15}$ For API identification and semi-quantitation by TLC, the sensitivity was $79 \%$ with $100 \%$ specificity. In Brazil, 14 of $46(30 \%)$ quinine samples were judged substandard by Minilab TLC with semi-quantitation. However, the seven samples that underwent confirmatory tests were all found to be within specifications. ${ }^{46}$ In the same study, all 289 samples collected in Guyana passed TLC with semi-quantitation, but 5 out of 10 samples failed subsequent confirmatory testing. A multicountry survey in Africa found that the Minilab detected $30 \%$ of 31 very non-compliant (deviation of $>20 \%$ from stated API by HPLC and/or percentage of API dissolved $>25 \%$ below the pharmacopeial limit $Q$ in dissolution testing) antimalarial samples. ${ }^{47}$ However, dissolution and disintegration tests measure different aspects of a solid formulation and we would not expect full agreement. TLC testing failed to 


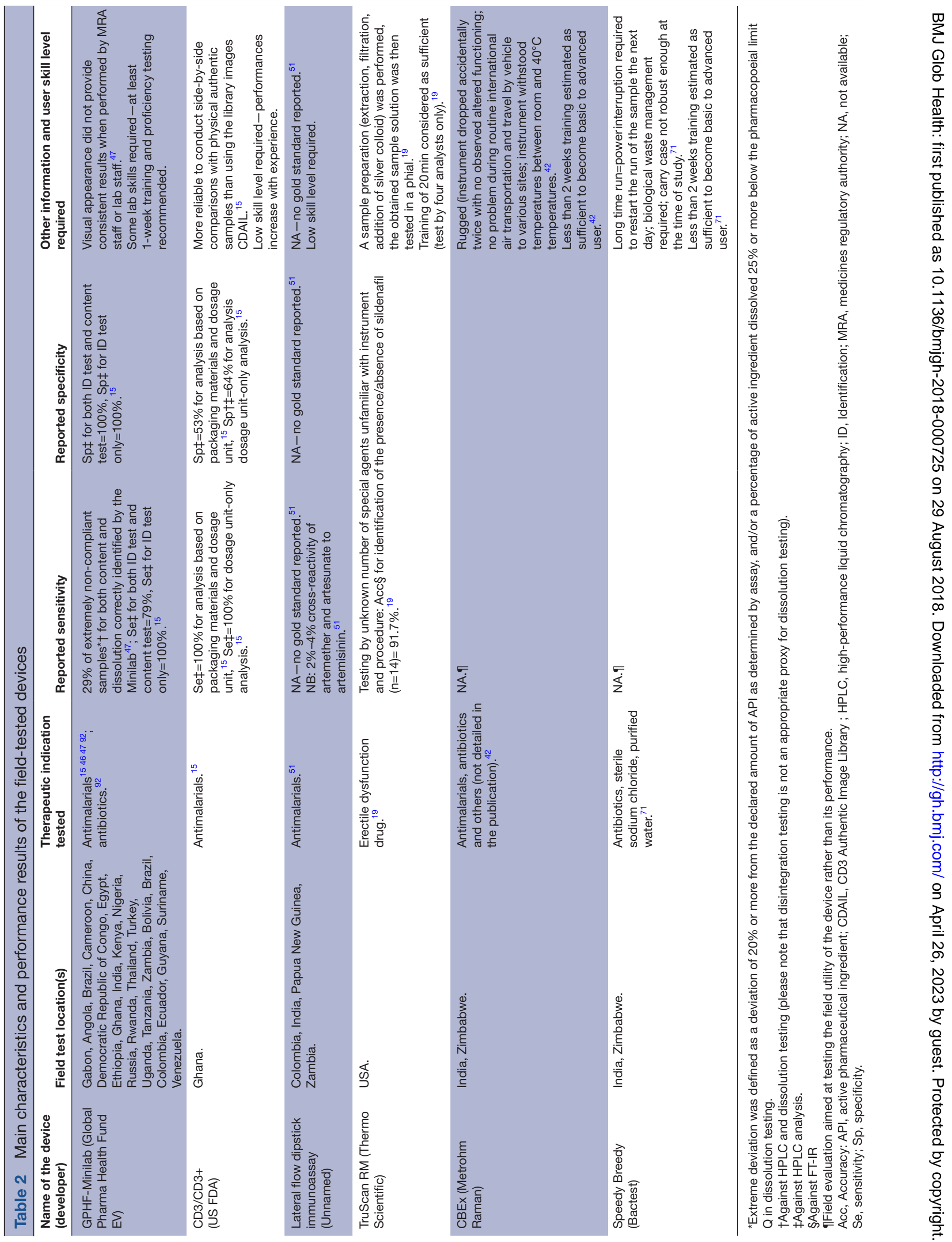


identify the 77 substandard antimicrobials, among which 76 samples contained \%API $>80 \%$ and $<123 \%$ of the label claim (specificity of 97\%) ${ }^{48}$ However, the Minilab is designed to detect samples with API below the $80 \%$ threshold. Interobserver variability was identified as a significant contributor to Minilab semi-quantitative inaccuracy. ${ }^{49}$ In Tanzania, seven drug inspectors assessed finished products containing four different APIs (antibiotics and antimalarials) at three different concentrations $(0 \%, 40 \%, 100 \%){ }^{49}$ Twenty-five out of 28 substandard samples with $40 \%$ API were incorrectly identified by TLC as of acceptable quality. After further training, 8 out of 28 samples were still wrongly identified as of acceptable quality. All samples with $100 \%$ API, zero API and with wrong API were correctly identified.

The PharmaChk is a field-portable microfluidics device, currently limited to artemisinin-based drugs, designed for API quantitation and tablet dissolution testing. ${ }^{50}$ Quantitation of three oral artesunate formulations (mean of five samples per formulation) showed accuracy to within $0 \%-4 \%$ of HPLC values. ${ }^{50}$

Single-use lateral flow immunoassay dipsticks (resembling rapid malaria diagnostic tests in appearance) use monoclonal antibodies to detect poor quality artemisinin-based antimalarials. ${ }^{51-53}$ Field survey samples (table 2 ) were not tested against a reference technique and hence sensitivity or specificity cannot be calculated. ${ }^{51}$ In laboratory testing, artesunate dipsticks showed $100 \%$ specificity for detecting artesunate against other commonly used antimalarials, including other artemisinin derivatives. ${ }^{53} \mathrm{~A}$ semi-quantitative analysis of API content was obtained by sample serial dilutions. ${ }^{53}$

A proof-of-concept paper describes the adaptation of the Fast Red TR reaction for artesunate detection onto paper-based microfluidic strips. ${ }^{54}$ The cards could detect the presence and determine relative concentration of artesunate in one genuine sample, and could detect its absence in two formulations containing artemether and dihydroartemisinin. Semi-quantitation accuracy was improved by greyscale intensity analysis using a smartphone app.

A battery-powered capillary electrophoresis device was able to successfully identify and quantify salbutamol and metoprolol in syrup and tablet formulations. Quantitation accuracy was within 3\%-13\% of results obtained by HPLC. ${ }^{55}$

Paper cards $(a P A D)$ have been successfully used for semi-quantitative iodometric back-titration of amoxicillin and ampicillin, tests specified in the pharmacopeial analysis of $\beta$-lactam antibiotics. ${ }^{56} 57$ These cards differentiated between amoxicillin solutions that varied in concentration by $0.15 \mathrm{mg} / \mathrm{mL}$, allowing identification of substandard amoxicillin $<83 \%$ of labelled API content. ${ }^{56}$ The aPADs gave errors of semi-quantitation of $13 \%$ and $5 \%$ (compared with HPLC) for 41 samples of amoxicillin and 40 samples of ampicillin collected in Kenya, respectively. ${ }^{57}$ In that study, aPADs identified samples containing below/above the US Pharmacopeia 90.0\% limit of the medicine stated \%API, with sensitivities of $73.2 \%$ and $80.0 \%$ for amoxicillin $(\mathrm{n}=80)$ and ampicillin $(\mathrm{n}=56)$, respectively (100\% specificities). The authors suggested that artificially degraded samples made for the purpose of the study (thermally stressed) may have led to decreased sensitivities.

By obtaining two-dimensional spectral data of tablets, an NIR imaging device (D-NIRS) could evaluate the distribution of different chemical components during tablet dissolution, ${ }^{58}$ but no sensitivity and specificity data of this device are available.

\section{Purely qualitative targeted API detection devices}

In contrast to the aPADs that enable semi-quantitation based on one chemical reaction (see above), the paper analytical devices (PADS) are designed for qualitative screening of APIs and some excipients. ${ }^{566} 61$ Separate lanes housing different colourimetric reactions produce a 'colour bar code', which is compared with a reference library of 'standard colour bar code images'. Expert readers can even discriminate different strengths of APIs. ${ }^{61}$

In testing of experimental formulations of known concentration, two antibiotics, three antituberculosis medicines and two antimalarials produced sensitivity values of $76 \%-100 \%(\mathrm{n}=9-60)$ and specificity of $80 \%-100 \%(\mathrm{n}=30-135) .{ }^{6061}$ The identification of APIs in coformulated samples was more variable. For example, in testing coformulated tuberculosis (TB) medicine samples, ethambutol was not detected when actually present in $30 \%$ and falsely reported as present when absent in $17 \%$ of tests. ${ }^{60}$ In testing 30 two-API coformulated TB samples, ethambutol and isoniazid were correctly detected in all samples. ${ }^{60}$

Two ion mobility spectrometry devices have been evaluated. $^{62}{ }^{63}$ The IONSCAN-LS (Smiths Detection) detected the APIs of erectile dysfunction drugs in 26 herbal supplements with $100 \%$ sensitivity and specificity, with successful identification of the specific API in 13 of $15(87 \%)$ samples. ${ }^{63}$ The SABRE 4000 (Smiths Detection) showed comparable results with a benchtop ion mobility instrument in detecting sibutramine in dietary supplements. ${ }^{62}$

\section{Devices which primarily examine physical properties}

Devices in this section primarily examine physical properties of the sample, such as their visual appearance. They cannot verify the presence or absence of the API. As falsified packaging is the key for identifying falsified medicines, they may have an important parallel functionality to chemical analysis devices.

\section{Visual/colour inspection}

The Counterfeit Detection Device CD3+ unmasks differences between test and authentic samples (packaging and dosage forms) by allowing the user to compare their appearance under diverse ultraviolet-visible and infrared (IR) wavelengths. ${ }^{1564-66}$ With this technique, falsified and 
genuine artesunate blister pack samples $(n=203)$ were identified with sensitivity and specificity of $98.4 \%$ and $100 \%$, respectively, with $100 \%$ interuser reliability. ${ }^{64}$ In a field study (table 2), 84 artemisinin-based combination therapies were identified with a sensitivity of $100 \%$ and specificities of $53 \%$ (on packaging materials and dosage unit) and $64 \%$ (on dosage unit only). ${ }^{15}$

The X-Rite Eye-One is an optical spectrometer that projects light of wavelengths $380-730 \mathrm{~nm}$ towards a solid surface, collects the reflected visible spectrum and digitally records it for comparison with a reference genuine sample. ${ }^{67}$ It correctly identified 40 out of 41 (98\%) samples of erectile dysfunction medicines, among which 35 were falsified. However, on testing genuine samples, $25 \%$ of packages and $15 \%$ of tablets were wrongly identified as falsified.

\section{Other physical properties}

Refractometry can be used to quantitatively detect APIs in solution by comparing the measured concentration with a concentration curve constructed from known standards. In testing whether an API was within $80 \%-120 \%$ of the stated concentration, the AR200 digital refractometer (Leica Microsystems) showed a sensitivity of $83 \%-100 \%$ and specificity of $56 \%-87 \%$ for 458 samples of 5 different poor quality antimalarials (both tablets and injectables) ${ }^{68}$

The Counterfeit Drug Indicator (CoDI) measures the ratio of laser light intensity transmitted and scattered on passing through a tablet, and compares the result from the test sample with that from an authentic tablet. The device correctly discriminated 6 falsified and 12 authentic artemether-lumefantrine tablets. ${ }^{65}$

The handheld SOC-410 (Surface Optics Corporation) uses directional hemispherical reflectance to analyse the surface of tablets in the mid-infrared and near-infrared range without the need for complex spectral interpretation. It showed $100 \%$ accuracy in the identification of one genuine and four falsified Viagra samples. ${ }^{69}$

A handheld gloss meter, based on diffractive optical elements, was developed to analyse differences in the magnitude of specular gloss of the surface of authentic and falsified tablets. The device results showed consistency with the findings from a two-dimensional gloss meter and an optical interference profilometer to screen for two falsified artemether-lumefantrine samples from Ghana. $^{70}$

\section{Microbial contamination detection}

The Speedy Breedy (Bactest), a portable respirometer that detects pressure changes as a proxy of microbial growth and hence contamination in liquid samples, showed sensitivities from $93.0 \%$ to $100 \%$ and specificities of $100 \%$ to identify microbial contamination by Escherichia coli of samples of sterile water for injection that were purposively spiked under various laboratory experimental conditions. Artesunate and oxytocin products for injection were correctly characterised for the presence/ absence of microbial contamination with E. coli and
Pseudomonas aeruginosa. Further evaluation of the field utility of the device (rather than its performance) showed that, despite the ability of the device to generate results under uncontrolled field settings (India and Zimbabwe), the requirement for a continuous power source during analysis (which can take more than a day) might be a barrier to its use in remote settings (table 2). ${ }^{71}$

\section{DISCUSSION}

The above results demonstrate the huge diversity of technologies and devices becoming available for the field detection and evaluation of medicines.

To maximise the detection and removal of poor quality medicines from the supply chain, a screening device with high sensitivity is required. Specificity is less vital as although low values would lead to additional work and cost in reference laboratory assays they would not lead to patient harm. Sensitivity data were found for few devices and were mostly derived from results of laboratory testing on a small number of samples of a few APIs. The median (range) number of APIs that were assessed per device was only 2 (1-20), a very meagre proportion of the $\sim 7000$ global international non-proprietary names of pharmaceutical substances. ${ }^{72}$

The increasing sophistication of falsified medicines requires advanced techniques that detect anomalies of packaging or product not apparent to the naked eye. Of the included devices, the CD3 and the X-Rite showed high sensitivity for packaging authenticity evaluation. The low-cost single-use technologies showed promise for qualitative analysis (PADs, lateral flow immunoassay dipsticks) and could be of great interest in the distal pharmaceutical supply chain. Very few devices have been evaluated for their ability to distinguish genuine from substandard medicines with reduced \%API. Most devices with the potential to assay API (semi)quantitatively in finished products require consumables and are destructive (GPHF-Minilab, PharmaChk, aPADs, lateral flow immunoassay dipsticks, paper-based microfluidic strip and capillary electrophoresis), except for spectroscopic devices. However, of the nine spectroscopic devices (TruScan, MIRA, EZRaman-I, MicroPhazir, MicroNIR, TruDefender, SCiO, QDa and Mini 10 mass spectrometer) tested for quantitation, none used automated methods, but required highly trained operators using complex API-specific calibration models, and are therefore not field-ready.

Tablet dissolution characteristics are key determinants of bioavailability and therefore efficacy. No marketed portable devices are currently able to evaluate dissolution, despite the likely contribution of poor dissolution antimicrobials to AMR. ${ }^{10}$ The under-development D-NIRS was the only portable device assessed for its ability to monitor dissolution and showed promising results, although on a limited number of samples. Methods for detecting other quality defects in substandard medicines, such as the presence of impurities or the lack of 
sterility, have received very little attention, except the Speedy Breedy which recently showed promise to identify microbial contamination in liquid samples. ${ }^{71}$ The distinction between degraded medicines, which left the factory with good quality but deteriorated due to poor storage and transport, and those failing due to errors in factory production is vital as the origins and solutions are different. The development of reference and screening API-specific technologies that could distinguish these issues will be of great importance.

Two-thirds of the devices $(27 / 41,66 \%)$ identified use spectroscopic techniques. Of these, only the TruScan and the NanoRam have been tested on a large number of samples in the laboratory. However, it was not possible to reliably and comprehensively review devices such as the TruScan for their performance, because one of the key publications could not be evaluated since the APIs contained in the products tested were not detailed. ${ }^{16}$ This emphasises the importance of ensuring that the databases of the results of device evaluations are made available to ensure their enduring value. A major advantage in an L/ MIC setting of many of the spectroscopic devices is the need for minimal end-user training, provided that the chemometrics analysis steps have already been bundled in user-friendly software. However, there are obstacles to their implementation. First, the purchase cost of most of these devices is high, likely prohibitively so, in L/MIC settings. User-friendly, miniaturised, low-cost spectrometers such as the SCiO (which can be operated using a smartphone) have recently shown promising performance. ${ }^{35}$ Second, the need for up-to-date reference specimens, whether as a prestored 'spectral reference library' or physical samples of quality-assured genuine medicines, adds significant work. ${ }^{73}$ 'In-built' libraries of raw materials available in some spectrometers are inappropriate for the screening of finished pharmaceutical products, as the spectra obtained are often influenced by both APIs and excipients and vary between brands. The difficulty of assembling quality-assured comparators and the need for frequent updating of stored spectral signatures may present a barrier to use unless the pharmaceutical industry efficiently and promptly provides updated samples or spectra when manufacturing processes change. Almost 30\% ( $n=114)$ of antimalarials collected in one study could not undergo Raman analysis because the authentic comparators could not be obtained by the investigators. ${ }^{43}$ Discussion on industry standards for spectra file format and transferability between devices using the same technology will be important.

Each spectroscopic technique has unique advantages and disadvantages. Using a combination of different spectroscopic techniques in parallel may be beneficial. For example, using a Raman spectrometer in combination with an IR spectrometer for tablets containing relatively low quantities of APIs may improve detection. ${ }^{16} 44$ Combining a spectroscopic tool with a visual inspection tool may also be synergistic. As far as we are aware, there have been no evaluations of combined technologies.
The widely distributed GPHF-Minilab (more than 800 units distributed in 95 countries) ${ }^{74}$ showed good performance in the identification of falsified medicines by TLC in one study and consistently high specificity. Results were user-dependent, underlying the importance of regular good quality training and proficiency testing. Of note, the disintegration testing in the Minilab kit is not an appropriate proxy for dissolution testing. The device showed limited ability to identify substandard medicines. In a recent study in China in which 77 samples were substandard, most being above the $80 \%$ API threshold in HPLC testing, $0 \%$ sensitivity (97\% specificity) was reported. ${ }^{48}$ However, vitally, the Minilab does not claim to be able to detect substandard medicines with API content above the $80 \%$ limit. Its main function has been to detect zero and wrong API medicines.

There are important limitations to this review. Our search included only scientific databases and only in English. We discounted 29 articles in which the stated aim was to develop, validate or compare chemometric techniques, rather than to assess the performance of the portable device itself, thereby excluding 13 portable devices (online supplementary file 7 ). This includes the Matrix-F (Bruker Optics), a non-handheld device used in over 300 mobile laboratories in China. ${ }^{75}$ Findings of device evaluation performed by non-independent evaluators risk bias and should be interpreted with caution.

Comparison between devices was significantly hindered by the heterogeneity of device evaluation methods and reporting. We found only two studies in which the Standards for Reporting Diagnostic Accuracy studies were followed. ${ }^{43}{ }^{48}$ Standardised guidance on how to assess and compare the performance of screening devices would be of great benefit. A recent article from the US Pharmacopoeial Convention addresses this. ${ }^{76}$ There is an urgent need for international organisations, the device and pharmaceutical industry and regulatory authorities to discuss norms and standards for medicine quality screening devices. In addition, field testing was conducted for just six devices, leaving a paucity of data on performance in the 'real-world'.

Other key gaps in the literature were identified (box 3). We observed a dire lack of information as to which medicines and formulations can be evaluated with each device. There has been a focus on anti-infective medicines (especially antimalarials), neglecting other medicine classes.

Chemical structures suggest a priori that some APIs will be problematic for certain devices. For example, NQR can only detect APIs with quadrupolar nuclei, such as ${ }^{14} \mathrm{~N}$. This is present in over $80 \%$ of medicines, ${ }^{77}$ but not, for example, the artemisinin derivatives. ${ }^{62}$ Similarly, some APIs, such as artesunate and quinine sulfate, have strong fluorescence with weak Raman scattering at $785 \mathrm{~nm}$, impairing the ability of such Raman devices to detect poor quality products labelled as containing these APIs. ${ }^{2078}$ Raman scattering from medicines with relatively low amounts of API(s) is often insufficient. ${ }^{164}$ Sensitivity of the PADs is also reduced for formulations with a low 


\section{Box 3 Key gaps in the literature}

Lack of independent evaluation of the majority of devices, particularly in field settings.

- Device performance tested on a very limited subset of available active pharmaceutical ingredients, predominantly anti-infectives.

- Very limited testing and comment on the ability of the devices to test through packaging and the type of packaging that is least obstructive to device use.

- Very limited comment on the inability of Raman or infrared spectroscopy to test capsules non-destructively, due to the opacity of capsule coating.

- Very limited information on the performance of devices to test liquid or parenteral formulations is available, with no data on testing of topical formulations.

- No studies looking at the effect of tablet coating on device performance.

- No information on cost-effectiveness.

- No testing or comment on the ability of the devices to distinguish between chiral enantiomers.

- Very limited comment on where in the pharmaceutical supply chain which devices are best employed.

- Very few studies which comment on training needs for accurate use of the devices.

proportion of API. ${ }^{61}$ More than half of pharmaceuticals are chiral compounds, with many enantiomers of racemic drugs showing marked differences in pharmacology. ${ }^{79} 80$ No discussion was found on the ability of the reviewed devices to discriminate different enantiomers. Theoretically only NQR would have this capability.

Most of the tested finished products in the included studies were tablets. Certain tablet coatings will likely provide a very difficult barrier to optical spectroscopic examination, as shown in testing of blue-coated dihydroartemisinin with the NanoRam. ${ }^{81}$ No data were found on testing of topical applications (eg, creams, gels) and little on liquids. It is unclear whether the devices lack the capability to test these formulations, or simply that this has not yet been investigated.

One vital but undiscussed issue is that (with the probable exception of $\mathrm{NQR}$ ) it will not be possible to non-destructively evaluate capsules unless spectroscopic techniques can be developed that allow the devices to 'see through' the capsule material. Consequently, a very sizeable proportion (in Laos, UK, France and USA, capsules comprise $11.4 \%,{ }^{82} 17.7 \%,{ }^{83} 9.7 \%{ }^{84}$ and $7.7 \%,{ }^{85}$ respectively, of registered oral medicines) of the global medicine supply will not be amenable to non-destructive spectroscopic evaluation. Non-destructive sampling was highlighted by different regulatory authority stakeholders as an ideal feature of a medicine quality screening device in a recent qualitative research paper. ${ }^{73}$ The use of transparent capsule shells could greatly expand IR or Raman devices utility. There are also few data on the ability of devices to accurately assess medicines through packaging (important when sample size is small and samples are required for legal investigations). How spectroscopic device accuracy changes with different types of glass and plastic packaging seems unknown. With such information, blister pack and tablet/capsule/powder/liquid bottle packaging could be designed to facilitate spectroscopic evaluation.

Further key aspects that have received minimal discussion include issues of device maintenance and quality assurance/quality control of the device performance (including calibration and performance quality checks).

The cost-effectiveness of introducing devices within postmarketing surveillance (PMS) systems, compared with other solutions for strengthening PMS, has not apparently been investigated. Screening technologies should be considered within a broader strategy to reduce the risk of poor quality medicines reaching patients. Given the substantial costs of using most of the devices in L/MICs, investment in devices should be compared with other strategies, such as enhancing inspection of manufacturing sites and evaluation of product dossiers. The public health effectiveness of detection of poor quality medicines will not be fulfilled unless reference laboratories are accessible and appropriate rapid responsive action is conducted.

How devices can be optimally used in different parts of the pharmaceutical supply has been little discussed, nor their integration into PMS. Their abilities may be overappreciated and vital routine visual packaging inspection reduced. Non-governmental organisations, procurement agencies and other institutions supplying medicines may also benefit from the use of reliable devices to check the quality of medicines they procure. Because those involved may have diverse educational backgrounds, data are needed to better understand the minimum level of training needed for appropriate use of the devices.

It seems unlikely, with current technology, that one device will be able to effectively monitor the quality of all medicines, and exploration of combinations of devices with different faculties is needed. The synergistic combination of devices with smartphones containing registration, batch number and packaging information for the country's medicines, and alerts of poor quality medicines in the region and to and from WHO holds great promise. As research expands on screening devices for testing different APIs, especially those coformulated, care will be needed with the public release of these data in order to avoid informing those making poor quality medicines of information that would allow them to circumvent detection of their 'products' by the screening devices.

For a small proportion of the globally available APIs, there is evidence that some devices will reliably detect falsified medicines, often containing zero or wrong API. However, there is much less evidence for their ability to detect substandard medicines, usually containing either too much, too little API or impaired dissolution. If such devices are used, it will be important to recognise this issue and not to regard a pass result as meaning that a medicine is good quality, only that it is not falsified. Clear statements from manufacturers and those evaluating 
these devices on their claimed capabilities and their limitations will be crucial to avoid overconfidence in their abilities.

\section{CONCLUSION}

The diversity of devices for medicines quality screening holds great hope for empowering medicine inspectors, making their work more cost-effective and actionable, and protecting patients from the harm of poor quality medicines. However, there is a vitally important lack of independent evaluation of the majority of devices, particularly in field settings. There is currently no device demonstrated to be able to screen the quality of all existing APIs available globally in different formulations and in different settings. Training and costs of implementing screening devices are major concerns, especially in L/MICs, but these considerations have not been explored. Intensive research is needed in order to provide the evidence national MRAs need to determine the optimal choice of device(s) to combat poor quality medicines.

Acknowledgements We are very grateful to the directors of Mahosot Hospital, the Director, Dr Manivanh Vongsouvath, and staff of the Microbiology Laboratory, Mahosot Hospital, all the developers/authors who responded to our queries regarding the devices and studies, and colleagues at the US Pharmacopeial Convention. We thank Dr Douglas Ball, Dr Susann Roth and Dr Sonalini Khetrapal for their support and advice. We also thank the reviewers of this paper for their useful feedback.

Contributors All the authors were involved in the conceptualisation and methodology of the study. CC, SV and MB designed the data collection tool. CC, SV $\mathrm{SZ}$ and $\mathrm{MB}$ were responsible for the formal analysis and investigation. CC, SV and $M B$ were responsible for the original draft preparation. FMF and PNN were involved in the supervision. All the authors equally contributed to the reviewing and editing of the manuscript.

Funding This work is funded under the work programme of the Regional Malaria and Other Communicable Disease Threats Trust Fund (RMTF), which was set up at the Asian Development Bank in December 2013, with the specific remit to support low-income and middle-income member countries to develop multicountry, crossborder and multisector responses to urgent malaria and other communicable disease issues. The RMTF's financing partners are the Government of Australia (Department of Foreign Affairs and Trade), the Government of Canada (Department of Foreign Affairs, Trade and Development), and the Government of the United Kingdom (Department for International Development). The funders had no role in study design, data collection and analysis, decision to publish, or preparation of the manuscript. The Wellcome Trust also funded CC, SV and PNN.

Competing interests None declared.

Patient consent Not required.

Provenance and peer review Not commissioned; externally peer reviewed.

Data sharing statement № additional data available.

Open access This is an open access article distributed in accordance with the Creative Commons Attribution 4.0 Unported (CC BY 4.0) license, which permits others to copy, redistribute, remix, transform and build upon this work for any purpose, provided the original work is properly cited, a link to the licence is given, and indication of whether changes were made. See: https://creativecommons.org/ licenses/by/4.0/.

\section{REFERENCES}

1. World Health Organization. A study on the public health and socioeconomic impact of substandard and falsified medical products. 2017. http://www.who.int/medicines/regulation/ssffc/
publications/Layout-SEstudy-WEB.pdf?ua=1 (accessed 15 Jan 2018).

2. Saunders W. Observations on the superior efficacy of the red Peruvian bark: in the cure of agues and other fevers. Interspersed with occasional remarks on the treatment of other diseases, by the same remedy, Ann Arbor: University of Michigan Library. 1782. http://name.umdl.umich.edu/004769880.0001.000 (accessed 1 Mar 2017).

3. Newton PN, Green MD, Fernández FM, et al. Counterfeit antiinfective drugs. Lancet Infect Dis 2006;6:602-13.

4. Tivura M, Asante I, van Wyk A, et al. Quality of artemisinin-based combination therapy for malaria found in Ghanaian markets and public health implications of their use. BMC Pharmacol Toxicol 2016:17:48.

5. Wafula F, Dolinger A, Daniels B, et al. Examining the quality of medicines at kenyan healthcare facilities: a validation of an alternative post-market surveillance model that uses standardized patients. Drugs Real World Outcomes 2017;4:53-63.

6. Kaur H, Clarke S, Lalani M, et al. Fake anti-malarials: start with the facts. Malar J 2016;15:86.

7. Securing Industry. Falsified packs of cancer drug Votrient found in Germany. 2017. https://www.securingindustry.com/pharmaceuticals/ falsified-cancer-drug-votrient-found-in-germany/s40/a3263/\#. WLacw2eL3MM (accessed 1 Mar 2017).

8. Securing Industry. Switzerland raises alarm over counterfeit Harvoni. 2016. https://www.securingindustry.com/pharmaceuticals/ switzerland-raises-alarm-over-counterfeit-harvoni-/s40/a2712/\#. WLadUGeL3MM (accessed 1 Mar 2017).

9. Securing Industry. More fake Harvoni found in Japan. 2017. https:// www.securingindustry.com/pharmaceuticals/more-fake-harvonifound-in-japan/s40/a3134/\#.WLadMWeL3MM (accessed 1 Mar 2017).

10. Newton PN, Caillet C, Guerin PJ. A link between poor quality antimalarials and malaria drug resistance? Expert Rev Anti Infect Ther 2016;14:531-3.

11. Przyswa E. Counterfeit medicines and criminal organizations. 2013. http://www.iracm.com/flip2/english/IRACM.html\#p=24 (accessed 2 May 2017).

12. Kovacs S, Hawes SE, Maley SN, et al. Technologies for detecting falsified and substandard drugs in low and middle-income countries. PLoS One 2014;9:e90601.

13. International Electrotechnical Commision. International Standard -IEC 60601-1. Medical electrical equipment. 2005. https://webstore. iec.ch/publication/2606

14. United States Pharmacopeia. USP reference standards - USP 38 General requirements (11). https://hmc.usp.org/sites/default/files/ documents/HMC/GCs-Pdfs/GC_pdf_USP38/c11.pdf (accessed 24 Aug 2017)

15. Batson JS, Bempong DK, Lukulay PH, et al. Assessment of the effectiveness of the CD3+ tool to detect counterfeit and substandard anti-malarials. Malar J 2016;15:119.

16. Dégardin K, Guillemain A, Roggo Y. Comprehensive study of a handheld Raman spectrometer for the analysis of counterfeits of solid-dosage form medicines. J Spectrosc 2017;2017:1-13.

17. Assi S, Watt R, Moffat T. Comparison of laboratory and handheld Raman instruments for the identification of counterfeit medicines. Spectroscopy 2011;36:46-7.

18. Luczak A, Kalyanaraman R. Portable and benchtop Raman technologies for product authentication and counterfeit detection. Am Pharm Rev 2014;17:76-80.

19. Lanzarotta A, Lorenz L, Batson JS, et al. Development and implementation of a pass/fail field-friendly method for detecting sildenafil in suspect pharmaceutical tablets using a handheld Raman spectrometer and silver colloids. J Pharm Biomed Anal 2017:146:420-5.

20. Hajjou M, Qin Y, Bradby S, et al. Assessment of the performance of a handheld Raman device for potential use as a screening tool in evaluating medicines quality. J Pharm Biomed Anal 2013;74:47-55

21. Kakio T, Yoshida N, Macha S, et al. Classification and visualization of physical and chemical properties of falsified medicines with handheld Raman spectroscopy and X-ray computed tomography. Am J Trop Med Hyg 2017;97:684-9.

22. Sorak D, Herberholz L, Iwascek S, et al. New developments and applications of handheld Raman, mid-infrared, and near-infrared spectrometers. Appl Spectrosc Rev 2012;47:83-115.

23. Lê LMM, Tfayli A, Zhou J, et al. Discrimination and quantification of two isomeric antineoplastic drugs by rapid and non-invasive analytical control using a handheld Raman spectrometer. Talanta 2016;161:320-4. 
24. Tondepu C, Toth R, Navin CV, et al. Screening of unapproved drugs using portable Raman spectroscopy. Anal Chim Acta 2017;973:75-81.

25. Lawson LS, Rodriguez JD. Raman barcode for counterfeit drug product detection. Anal Chem 2016;88:4706-13.

26. Degardin K, Roggo Y, Margot P. Evaluation of Raman, infrared and near infrared hand held spectrometers for the detection of counterfeit medicines. Spectra Anal 2010;39:46-52.

27. Bate R, Tren R, Hess K, et al. Pilot study comparing technologies to test for substandard drugs in field settings. Afr J Pharm Pharmacol 2009;3:165-70.

28. Kalyanaraman R, Dobler G, Ribick M. Portable spectrometers for pharmaceutical counterfeit detection. Am Pharm Rev 2010;13:38-45.

29. Ma B, Wang L. An application of rapid detection technologies in a national regulatory laboratory setting: Differentiating imported and domestic drug products of oxcarbazepine using handheld Raman, near infrared, and portable FTIR analyzers. Am Pharm Rev 2015;18.

30. Ma B, Huong LTT, Liu Y, et al. Rapid detection of counterfeit drugs of ethambutol hydrochloride and cefuroxime axetil using handheld Raman, near infrared and portable FTIR technologies. Am Pharm Rev 2014;17:1-21.

31. Zontov YV, Balyklova KS, Titova AV, et al. Chemometric aided NIR portable instrument for rapid assessment of medicine quality. $J$ Pharm Biomed Anal 2016;131:87-93.

32. Obeidat SM, AL-TAYYEM BAN. Spectroscopic and chemometric analysis of illegally manufactured formulations of selected medicines. Orient J Chem 2012;28:795-801.

33. Polli JE, Hoag SW, Flank S. Near-infrared spectrophotometric comparison of authentic and suspect pharmaceuticals. Pharm Technol 2009;33:46-52.

34. Alcalà $M$, Blanco $M$, Moyano $D$, et al. Qualitative and quantitative pharmaceutical analysis with a novel hand-held miniature near infrared spectrometer. J Near Infrared Spectrosc 2013;21:445-57.

35. Wilson BK, Kaur H, Allan EL, et al. A New handheld device for the detection of falsified medicines: demonstration on falsified artemisinin-based therapies from the field. Am J Trop Med Hyg 2017:96:1117-23

36. Bernier MC, Li F, Musselman B, et al. Fingerprinting of falsified artemisinin combination therapies via direct analysis in real time coupled to a compact single quadrupole mass spectrometer. Anal Methods 2016;8:6616-24.

37. Keil A, Talaty N, Janfelt C, et al. Ambient mass spectrometry with a handheld mass spectrometer at high pressure. Anal Chem 2007;79:7734-9.

38. Sacré PY, Deconinck E, Saerens L, et al. Detection of counterfeit Viagra ${ }^{\circledR}$ by Raman microspectroscopy imaging and multivariate analysis. J Pharm Biomed Anal 2011;56:454-61.

39. Corrigan DK, Salton NA, Preston C, et al. Towards the development of a rapid, portable, surface enhanced Raman spectroscopy based cleaning verification system for the drug nelarabine. J Pharm Pharmacol 2010;62:1195-200.

40. Fukami T, Koide T, Hisada $\mathrm{H}$, et al. Pharmaceutical evaluation of atorvastatin calcium tablets available on the Internet: A preliminary investigation of substandard medicines in Japan. J Drug Deliv Sci Technol 2016;31:35-40.

41. Kindenge JM, Tshilombo NK, Chavez P-F, et al. Near infrared spectroscopy, a non-destructive technique to fight against counterfeit medicines. Spectra Anal 2014;43:43-6.

42. United States Pharmacopoeial Convention. USP technology review: CBEx. 2017. http://www.usp.org/sites/default/files/usp/document/ our-work/global-public-health/tr-report-cbex.pdf (accessed 8 May 2018).

43. Visser BJ, de Vries SG, Bache EB, et al. The diagnostic accuracy of the hand-held Raman spectrometer for the identification of antimalarial drugs. Malar J 2016;15:160/1-160/12.

44. Assi S. Investigating the quality of medicines using handheld Raman spectroscopy. Eur Pharm Rev 2014;19:56-60.

45. Barras J, Katsura S, Sato-Akaba H, et al. Variable-pitch rectangular cross-section radiofrequency coils for the nitrogen-14 nuclear quadrupole resonance investigation of sealed medicines packets. Anal Chem 2012;84:8970-2.

46. Pribluda VS, Barojas A, Añez A, et al. Implementation of basic quality control tests for malaria medicines in Amazon Basin countries: results for the 2005-2010 period. Malar J 2012;11:202.

47. World Health Organization. Survey of the quality of Selected antimalarial medicines circulating in Six countries of SubSaharan africa, Geneva, Switzerland. 2011. http://apps.who.int/ medicinedocs/en/d/Js17835en/ (accessed 28 Aug 2017).
48. Pan H, Ba-Thein W. Diagnostic accuracy of Global Pharma Health Fund Minilab ${ }^{T M}$ in assessing pharmacopoeial quality of antimicrobials. Am J Trop Med Hyg 2017:tpmd170289.

49. Risha P, Msuya Z, Ndomondo-Sigonda M, et al. Proficiency testing as a tool to assess the performance of visual TLC quantitation estimates. J AOAC Int 2006;89:1300-4.

50. Desai D. PharmaChk: Robust device for counterfeit and substandard medicines screening in developing regions. 2014. https://open.bu. edu/handle/2144/12087

51. Guo S, He L, Tisch DJ, et al. Pilot testing of dipsticks as point-ofcare assays for rapid diagnosis of poor-quality artemisinin drugs in endemic settings. Trop Med Health 2016;44:15.

52. He L, Nan T, Cui Y, et al. Development of a colloidal gold-based lateral flow dipstick immunoassay for rapid qualitative and semiquantitative analysis of artesunate and dihydroartemisinin. Malar $J$ 2014;13:127

53. Guo S, Zhang W, He L, et al. Rapid evaluation of artesunate quality with a specific monoclonal antibody-based lateral flow dipstick. Anal Bioanal Chem 2016;408:6003-8.

54. Koesdjojo MT, Wu Y, Boonloed A, et al. Low-cost, high-speed identification of counterfeit antimalarial drugs on paper. Talanta 2014;130:122-7.

55. Nguyen TA, Pham TN, Doan TT, et al. Simple semi-automated portable capillary electrophoresis instrument with contactless conductivity detection for the determination of $\beta$-agonists in pharmaceutical and pig-feed samples. J Chromatogr $A$ 2014;1360:305-11.

56. Myers NM, Kernisan EN, Lieberman M. Lab on paper: iodometric titration on a printed card. Anal Chem 2015;87:3764-70.

57. Myers N. Lab on a paper: adapting quantitative chemical techniques for use in low resource areas. 2017. https://curate.nd.edu/ downloads/und:m900ns08m33 (accessed 9 May 2018).

58. Ishikawa D, Murayama K, Awa K, et al. Application of a newly developed portable NIR imaging device to monitor the dissolution process of tablets. Anal Bioanal Chem 2013;405:9401-9.

59. Ishikawa D, Murayama K, Genkawa T, et al. Development of a compact near infrared imaging device with high-speed and portability for pharmaceutical process monitoring. NIR news 2012;23:14-17.

60. Weaver AA, Reiser H, Barstis T, et al. Paper analytical devices for fast field screening of beta lactam antibiotics and antituberculosis pharmaceuticals. Anal Chem 2013;85:6453-60.

61. Weaver AA, Lieberman M. Paper test cards for presumptive testing of very low quality antimalarial medications. Am J Trop Med Hyg 2015;92:17-23.

62. Dunn JD, Gryniewicz-Ruzicka CM, Kauffman JF, et al. Using a portable ion mobility spectrometer to screen dietary supplements for sibutramine. J Pharm Biomed Anal 2011;54:469-74.

63. Gryniewicz CM, Reepmeyer JC, Kauffman JF, et al. Detection of undeclared erectile dysfunction drugs and analogues in dietary supplements by ion mobility spectrometry. J Pharm Biomed Anal 2009;49:601-6.

64. Ranieri N, Tabernero P, Green MD, et al. Evaluation of a new handheld instrument for the detection of counterfeit artesunate by visual fluorescence comparison. Am J Trop Med Hyg 2014;91:920-4.

65. Green MD, Hostetler DM, Nettey $\mathrm{H}$, et al. Integration of novel low-cost colorimetric, laser photometric, and visual fluorescent techniques for rapid identification of falsified medicines in resourcepoor areas: application to artemether-lumefantrine. Am J Trop Med Hyg 2015;92:8-16.

66. Lanzarotta A, Ranieri N, Albright D, et al. Analysis of counterfeit FDA-Regulated products at the forensic chemistry center: Rapid visual and chemical screening procedures inside and outside of the laboratory. Am Pharm Rev 2015;18.

67. Rodomonte AL, Gaudiano MC, Antoniella E, et al. Counterfeit drugs detection by measurement of tablets and secondary packaging colour. J Pharm Biomed Anal 2010;53:215-20.

68. Green MD, Nettey H, Villalva Rojas O, et al. Use of refractometry and colorimetry as field methods to rapidly assess antimalarial drug quality. J Pharm Biomed Anal 2007:43:105-10.

69. Wilczyński S, Koprowski R, Błońska-Fajfrowska B. Directional reflectance analysis for identifying counterfeit drugs: Preliminary study. J Pharm Biomed Anal 2016;124:341-6.

70. Bawuah P, Pääkkönen P, Peiponen K-E. Gloss measurement in detection of surface quality of pharmaceutical tablets: a case study of screening of genuine and counterfeit antimalaria tablets. J Eur Opt Soc Publ 2017;13:18.

71. United States Pharmacopoeial Convention. USP technology review: Speedy Breedy. http://www.usp.org/sites/default/files/usp/ document/our-work/global-public-health/technology-review-reportspeedy-breedy.pdf (accessed 8 May 2018). 
72. World Health Organization. Guidance on INN. http://www.who.int/ medicines/services/inn/innquidance/en/ (accessed 3 Jul 2017).

73. Roth L, Nalim A, Turesson B, et al. Global landscape assessment of screening technologies for medicine quality assurance: stakeholder perceptions and practices from ten countries. Global Health 2018;14:43.

74. Global Pharma Health Fund E.V. GPHF-MinilabTM - fact sheet: Global Pharma Health Fund E.V. (n.d.). GPHF-Minila. https://www. gphf.org/en/minilab/factsheet.htm (accessed 30 Jun 2017).

75. Feng $\mathrm{Y}-\mathrm{C}, \mathrm{Hu} \mathrm{C}-\mathrm{Q}$, Yang $\mathrm{XL}$, et al. Monitoring the quality of drugs in circulation using rapid NIR spectral comparison methods. J Chinese Pharm Sci 2011;20:290-6.

76. United States Pharmacopoeial Convention. General chapter prospectus: evaluation of screening technologies for assessing medicines quality. 2017. http://www.uspnf.com/notices/evaluatingscreening-technologies-for-assessing-medicine-quality

77. Barras J, Kyriakidou G, Poplett IJF, et al. The nuclear quadrupole resonance-based screening of medicines. 2012;3930:5576.

78. Ricci C, Nyadong L, Yang F, et al. Assessment of hand-held Raman instrumentation for in situ screening for potentially counterfeit artesunate antimalarial tablets by FT-Raman spectroscopy and direct ionization mass spectrometry. Anal Chim Acta 2008;623:178-86.

79. Nguyen LA, He H, Pham-Huy C. Chiral drugs: an overview. Int J Biomed Sci 2006;2:85-100.

80. Caillet C, Chauvelot-Moachon L, Montastruc JL, et al. Safety profile of enantiomers vs. racemic mixtures: it's the same? $\mathrm{Br} J$ Clin Pharmacol 2012;74:886-9.

81. Visser BJ, Meerveld-Gerrits J, Kroon D, et al. Assessing the quality of anti-malarial drugs from Gabonese pharmacies using the MiniLab®: a field study. Malar J 2015;14:273.

82. Food and drug department; Ministry of Health; Lao PDR. List of registered medicines. 2017. http://www.fdd.gov.la/showContent_en. php?contID=32 (accessed 28 Aug 2017).

83. Joint Formulary Committee. British National Formulary. BMJ group and pharmaceutical press. 2017. https://www.medicinescomplete. com/mc/bnf/current/index.htm
84. Agence Nationale de Sécurité du Médicament et des Produits de Santé. Répertoire des Spécialités Pharmaceutiques. Fich. des spécialités. 2017. http://agence-prd.ansm.sante.fr/php/ecodex/ telecharger/telecharger.php (accessed 10 Oct 2017).

85. United States Food and Drug Administration. National Drug Code Directory. 2017. https://www.fda.gov/drugs/informationondrugs/ ucm142438.htm (accessed 4 Oct 2017).

86. SF Medical Products Group, Essential Medicines and Health Products WHO. WHO Member State Mechanism on Substandard/Spurious/Falsely-Labelled/Falsified/Counterfeit (SSFFC) Medical Products. In: seventieth world health assembly. Geneva, Switzerland: 2017. A70/23. http://www.who.int/ medicines/regulation/ssffc/A70_23-en1.pdf?ua=1

87. Bate R, Hess K. Anti-malarial drug quality in Lagos and Accra - a comparison of various quality assessments. Malar J 2010;9:157.

88. Kalyanaraman R, Ribick M, Dobler G. Portable Raman spectroscopy for pharmaceutical counterfeit detection. Eur Pharm Rev 2012;17:11-15.

89. Polli JE, Hoag SW, Flank S. Near-infrared spectrophotometric: comparison of authentic and suspect pharmaceuticals. Pharm Technol 2009;33:46-52.

90. Pederson CG, Friedrich DM, Hsiung C, et al. Pocket-size nearinfrared spectrometer for narcotic materials identification. In: Nextgeneration spectroscopic technologies VII, Proceedings of SPIE, 2014;9101:910100-1-11.

91. Bate R, Jensen P, Hess K, et al. Substandard and falsified antituberculosis drugs: a preliminary field analysis. Int J Tuberc Lung Dis 2013;17:308-11.

92. Risha PG, Msuya Z, Clark M, et al. The use of Minilabs to improve the testing capacity of regulatory authorities in resource limited settings: Tanzanian experience. Health Policy 2008;87:217-22.

93. Bawuah P, Pääkkönen P, Peiponen K-E. Gloss measurement in detection of surface quality of pharmaceutical tablets: a case study of screening of genuine and counterfeit antimalaria tablets. AcadRapid Publ 2017;13:18. 